\title{
Eigenvalues and eigenfunctions of the Laplacian via inverse iteration with shift
}

\author{
Rodney Josué Biezuner ${ }^{\mathrm{a}}$, Grey Ercole ${ }^{\mathrm{a}, *}$, Breno Loureiro Giacchini ${ }^{\mathrm{b}}$, Eder Marinho Martins ${ }^{\mathrm{c}}$ \\ a Departamento de Matemática-ICEx, Universidade Federal de Minas Gerais, Av. Antônio Carlos 6627, Caixa Postal 702, 30161-970 Belo Horizonte, MG, Brazil \\ ${ }^{\mathrm{b}}$ Departamento de Física-ICEx, Universidade Federal de Minas Gerais, Av. Antônio Carlos 6627, 31270-901 Belo Horizonte, MG, Brazil \\ ${ }^{\mathrm{c}}$ Departamento de Matemática-ICEB, Universidade Federal de Ouro Preto, Campus Universitário Morro do Cruzeiro, $35400-000$ Ouro Preto, MG, Brazil
}

\section{A R T I C L E I N F O}

\section{Keywords:}

Laplacian

Eigenvalues

Eigenfunctions

Fourier series

Inverse iteration with shift

Rayleigh quotient

\begin{abstract}
A B S T R A C T
In this paper we present an iterative method, inspired by the inverse iteration with shift technique of finite linear algebra, designed to find the eigenvalues and eigenfunctions of the Laplacian with homogeneous Dirichlet boundary condition for arbitrary bounded domains $\Omega \subset \mathbb{R}^{N}$. This method, which has a direct functional analysis approach, does not approximate the eigenvalues of the Laplacian as those of a finite linear operator. It is based on the uniform convergence away from nodal surfaces and can produce a simple and fast algorithm for computing the eigenvalues with minimal computational requirements, instead of using the ubiquitous Rayleigh quotient of finite linear algebra. Also, an alternative expression for the Rayleigh quotient in the associated infinite dimensional Sobolev space which avoids the integration of gradients is introduced and shown to be more efficient. The method can also be used in order to produce the spectral decomposition of any given function $u \in L^{2}(\Omega)$.
\end{abstract}

(c) 2012 Elsevier Inc. All rights reserved.

\section{Introduction}

In [1] we introduced an iterative method for computing the first eigenpair of the $p$-Laplacian operator $\Delta_{p} u:=\operatorname{div}\left(|\nabla u|^{p-2} \nabla u\right), p>1$, with homogeneous Dirichlet boundary condition in a bounded domain $\Omega \subset \mathbb{R}^{N}, N \geqslant 1$. The technique was inspired by the inverse power method or inverse iteration of finite linear algebra.

In the present paper we concentrate in the special case $p=2$, the Laplace operator $\Delta$, which was superficially dealt with in [1]. Besides clarifying some of the arguments sketched in that paper for this case and providing some error estimates, our main purpose in this work is to show how inverse iteration with shift in the presence of uniform convergence can be used in order to obtain a fast and efficient method for computing the eigenvalues and eigenfunctions of the Laplacian operator with homogeneous Dirichlet boundary condition for any bounded domain $\Omega$. If the eigenvalues or at least good estimates for them are a priori known, the method can produce the corresponding eigenfunctions with great speed and accuracy.

The technique can alternatively also be used as a fast process to obtain the spectral decomposition of any function $u \in L^{2}(\Omega)$ (in other words, the Fourier series of $u$ ).

We remark that the application of the method to the special case of the Laplacian operator is more natural since the Laplacian is a linear operator, $L^{2}(\Omega)$ is a Hilbert space and the inverse operator $-\Delta^{-1}$ is a self-adjoint and compact operator, therefore allowing the complete characterization of its spectral structure, as well as having the property that its eigenfunctions constitute a basis for $L^{2}(\Omega)$ (except for compactness, these properties are absent in the $p$-Laplacian when $p \neq 2$ ).

\footnotetext{
* Corresponding author.

E-mail addresses: rodney@mat.ufmg.br (R.J. Biezuner), grey@mat.ufmg.br (G. Ercole), brenolg@ufmg.br (B.L. Giacchini), eder@iceb.ufop.br (E.M. Martins).
} 
Our approach of the inverse iteration with shift is based on the following iterative process started by a given function $u \in L^{2}(\Omega)$ :

$$
\phi_{0}:=u \quad \text { and } \begin{cases}-\Delta_{\sigma} \phi_{n+1}=\phi_{n} & \text { in } \Omega, \\ \phi_{n+1}=0 & \text { on } \partial \Omega\end{cases}
$$

where $\sigma>0$ is a previously fixed shift and $\Delta_{\sigma}:=\Delta+\sigma I$ is the corresponding shifted operator.

The sequence $\left\{\phi_{n}\right\}$ is then handled in order to produce approximations for the pair $\left(\lambda_{u}^{\sigma}, e_{u}^{\sigma}\right)$ where $\lambda_{u}^{\sigma}$ denotes the eigenvalue of the Laplacian appearing in the spectral expansion of $u$ which is closest to $\sigma$, and $e_{u}^{\sigma}$ denotes the eigenfunction obtained as the projection of $u$ on the $\lambda_{u}^{\sigma}$-eingenspace.

Inverse iteration with shift is used in finite linear algebra in order to find the eigenvalues and eigenfunctions of a finitedimensional linear operator. As an eigenvalue-finding procedure it is not as efficient as other methods, such as the $Q R$ algorithm. However, if very good estimates of the eigenvalues are known in advance, its rate of convergence to both eigenvalues and eigenfunctions can be very fast (see [2], for instance).

This approach can be naturally extended to self-adjoint compact linear operators in infinite-dimensional Hilbert spaces such as the Laplacian and those arising in Sturm-Liouville problems. In spite of this, we have not been able to find any reference in the literature to this approach being used in the Laplacian context.

Since there is now a vast literature concerning the search for estimates for the eigenvalues of the Laplacian, as well as the gaps between eigenvalues (see, for instance, [3-6]; although particularly useful in our context would be lower bounds for the difference between consecutive eigenvalues), these results can be used in connection with the inverse iteration with shift algorithm to find eigenfunctions of the Laplacian in arbitrary domains, as well as better approximations for its eigenvalues. It must be emphasized, however, that as with the finite linear method, the inverse iteration with shift method is not capable to find all the eigenfunctions associated to a non-simple eigenvalue. It can only find an eigenfunction of the associated eigenspace. In the generic sense most domains have Laplacian spectra consisting only of simple eigenvalues (see [7,8]), although many domains of practical interest have eigenvalues with multiplicity greater than one (usually, domains which exhibit some type of symmetry, although not all of them).

Algorithm 1 below is the simplest version of the inverse iteration with shift algorithm for computing one specific eigenvalue and corresponding eigenfunction of the Laplacian.

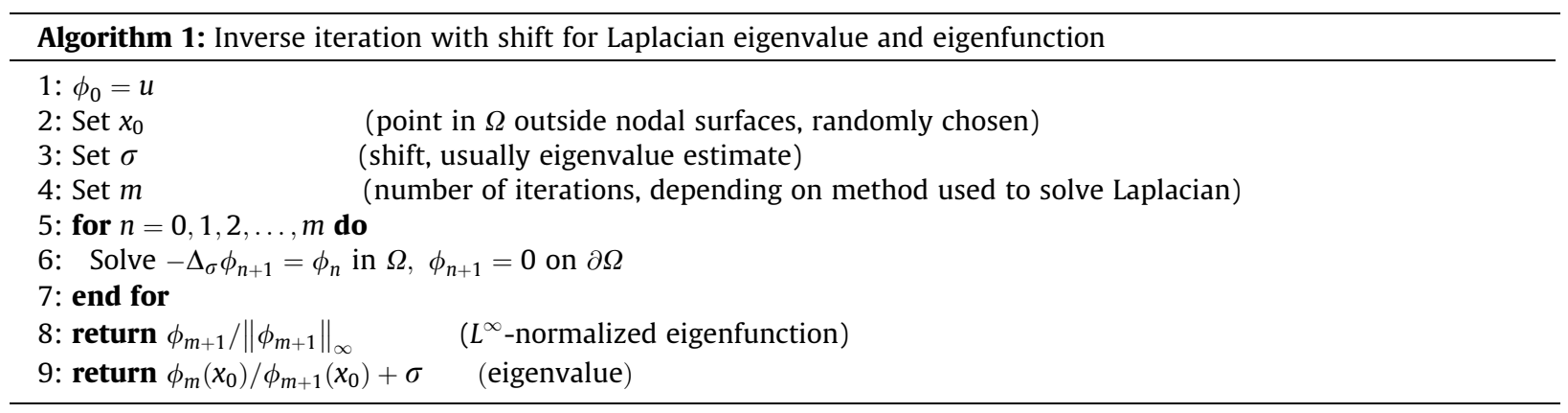

In principle, the function $u$ at the start of Algorithm 1 should be chosen so that it will have components in all eigenspaces of the Laplacian and a random choice would suffice. However, in practice, due to rounding errors any simple function can be used. In our numerical tests (see Section 6), we observed that even the unit constant function could be used in order to obtain all the eigenvalues, even in a domain where it does not have an infinite number of them in its spectral decomposition.

In Line 6 of Algorithm 1 any PDE solver can be used. This allows one to choose the fastest solver available for a particular domain. In Line 9 the eigenvalue is computed according to the uniform convergence theory developed in Section 4 . Since uniform convergence occurs away from nodal surfaces, a point $x_{0} \in \Omega$ not in a nodal surface must be chosen; because nodal surfaces have zero $\mathrm{N}$-dimensional measures, a random choice will suffice in the vast majority of cases, even taking into account that nodal surfaces change as the computed eigenfunction changes.

In finite linear algebra, the approximation to the eigenvalue is usually computed using the Rayleigh quotient. In our context, the eigenvalue can also be computed via the Rayleigh quotient of the approximated eigenfunction:

$$
\mathcal{R}\left(\phi_{n+1}\right)=\frac{\left\langle\nabla \phi_{n+1}, \nabla \phi_{n+1}\right\rangle_{2}}{\left\langle\phi_{n+1}, \phi_{n+1}\right\rangle_{2}}=\frac{\left\|\nabla \phi_{n+1}\right\|_{2}^{2}}{\left\|\phi_{n+1}\right\|_{2}^{2}}=\frac{\int_{\Omega}\left|\nabla \phi_{n+1}\right|^{2} d x}{\int_{\Omega} \phi_{n+1}^{2} d x} .
$$

However, due to the high oscillatory nature of high frequency eigenfunctions, in order to accurately compute the integral of the (squared) gradient of eigenfunctions associated with these eigenvalues, a much finer grid needs to be used, which affects the efficiency of the method. Therefore, the Rayleigh quotient in its original form (2) is not recommended for the computation of the eigenvalues of the Laplacian, unless one is prepared to incur the higher computational costs (see also further comments in Section 7). 
Nonetheless, an integration by parts argument produces the following alternative expression for the Rayleigh quotient that avoids computations involving gradients:

$$
\mathcal{R}\left(\phi_{n}\right)=\sigma+\frac{\int_{\Omega} \phi_{n} \phi_{n-1} d x}{\int_{\Omega}\left|\phi_{n}\right|^{2} d x} .
$$

We obtain very satisfactory results by using this sequence in Line 9 of Algorithm 1 to compute eingenvalues in our numerical tests (see Section 6).

Another alternative way to compute the eigenvalues is given by the quotient

$$
\frac{\left\|\phi_{n}\right\|_{2}}{\left\|\phi_{n+1}\right\|_{2}}+\sigma=\frac{\int_{\Omega} \phi_{n}^{2} d x}{\int_{\Omega} \phi_{n+1}^{2} d x}+\sigma,
$$

when the shift $\sigma$ is chosen below the eigenvalue. This quotient also gives accurate approximations for the eigenvalues even using relatively coarse meshes; the computation of the integrals of the approximated eigenfunctions, instead of their gradients, does not appear to be significantly affected by the use of coarse grids (see Section 6).

We believe that, differently from what happens in finite dimensions, where much better and faster algorithms for finding the eigenvalues of linear operators (matrices), specially self-adjoint operators, are available, the inverse iteration with shift algorithm can be a very competitive method for finding the eigenvalues of the Laplacian. Typical algorithms for computing Laplacian eigenvalues involve the discretization of the Laplacian operator and the computation of the eigenvalues of the resulting discretization matrix. However, since only a small number of the eigenvalues of the discretization matrix are good approximations to the Laplacian eigenvalues (the smaller ones), huge matrices are necessary in order to obtain a sufficiently good number of eigenvalues. And some problems, particularly those arising in the study of quantum billiards, demand the computation of a very large number of eigenvalues. Needless to say, besides the requirements of memory, the size of the matrix makes it computationally costly to find its eigenvalues (see the classical [9] book, the review [3] and the more recent work [10] for details).

The inverse iteration with shift method, that requires only typical relatively modest sizes for meshes in order to solve the Poisson equation with homogeneous Dirichlet boundary condition, can be very competitive in terms of memory allocation and processing time. This is true even when one considers that in order to find accurate approximations for the highest order eigenvalues and eigenfunctions sometimes one needs to refine the mesh, due to the increase of oscillations.

Even if good estimates for the eigenvalues of a particular domain are not known in advance, a few iterations of inverse iteration with shift should be able to find good approximations to them, which can work as first estimates for the shift on a second run of the algorithm. The first choices for the shift might be concentrated around the numbers given by Weiyl's Law (see [11] or [12]).

The inverse iteration with shift method can also be used in order to find the spectral decomposition of any function $u \in L^{2}(\Omega)$, that is, in order to find its projections on the Laplacian eigenspaces. One has only to be careful to eliminate spurious projections, that is, projections which arise from rounding errors. This can be done through computing the Fourier coefficient associated to each eigenspace. If this coefficient becomes less than a specified very small tolerance, this projection can be safely discarded as arising from rounding errors. The two algorithms can be combined together in order to simultaneously find both the desired spectral decomposition of a given function defined on a domain and the spectrum of the Laplacian on it. The spectral decomposition algorithm is given below (Algorithm 2). Once again, Line 11 can be replaced by (3) or (4).

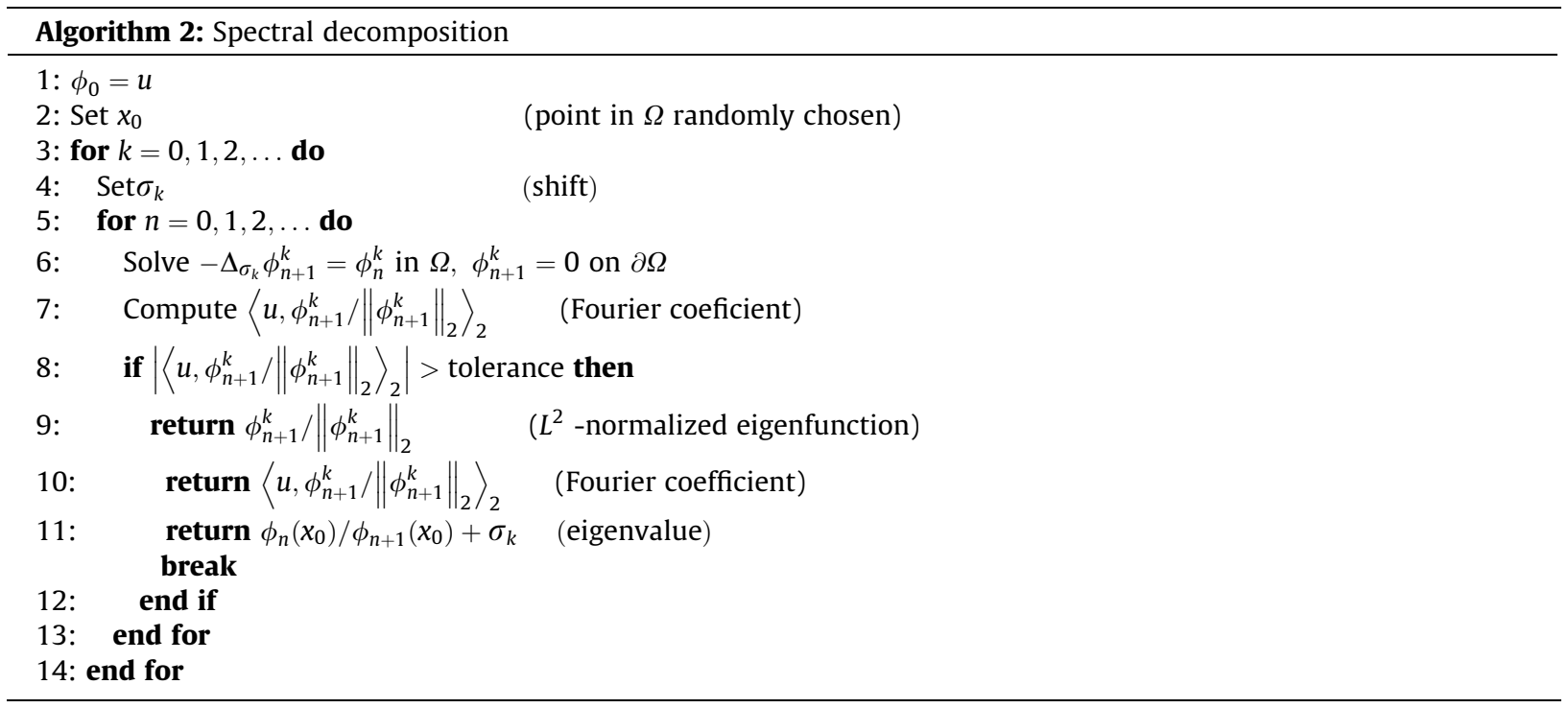


Although for the sake of simplicity all computations here are done for the Laplacian, the same algorithm can be used for similar elliptic operators.

This paper is organized as follows. The inverse iteration with shift sequence is defined in Section 2, where most of the notation used in this paper is also established. In Section 3 some well-known results concerning the Rayleigh quotient are recalled and proven for completeness. In Sections 3 and 4 we discuss $L^{2}$ and uniform convergence of the inverse iterated sequence, respectively. In the short Section 5 we make some considerations about the normalization process on each step of (1) which is useful for computational purposes, and in Section 6 we present the results of some numerical experiments made in simple domains.

Finally, in Section 7 we discuss if the rate of convergence of the method could theoretically be improved through the use of the inverse iteration with shift given by the Rayleigh quotient, as it is standard practice in finite linear algebra.

\section{Definition of the inverse iteration with shift sequence}

Let $\Omega$ be a bounded domain in $\mathbb{R}^{N}$ and $\mathcal{H}=\left\{e_{k}\right\}_{k=1}^{\infty} \subset H_{0}^{1}(\Omega)$ be an orthogonal (not necessarily normalized) basis for $L^{2}(\Omega)$ consisting of eigenfunctions of the Laplacian operator with homogeneous Dirichlet boundary condition, that is,

$$
\begin{cases}-\Delta e_{k}=\lambda_{k} e_{k} & \text { in } \Omega, \\ e_{k}=0 & \text { on } \partial \Omega,\end{cases}
$$

where $\left\{\lambda_{k}\right\}_{k=1}^{\infty}$ is the non-decreasing sequence of eigenvalues of the Laplacian, counting multiplicities:

$$
0<\lambda_{1}<\lambda_{2} \leqslant \ldots
$$

Let $\sigma>0$ and define the shifted operator

$$
\Delta_{\sigma}:=\Delta+\sigma I \text {. }
$$

It follows that $e_{k}$ is also an eigenfunction of $-\Delta_{\sigma}$ corresponding to the eigenvalue $\lambda_{k}-\sigma$.

Conversely, if $\lambda$ is an eigenvalue of $-\Delta_{\sigma}$, then $\lambda=\lambda_{k}-\sigma$ for some $k$. Thus, the spectrum of the shifted operator equals the spectrum of the Laplacian operator shifted to the left by $\sigma$, while the corresponding eigenspaces are the same.

Given $u \in L^{2}(\Omega)$, let

$$
u=\sum_{k=1}^{\infty} \alpha_{k} e_{k}
$$

be the Fourier expansion of $u$, so that the Fourier coefficients $\alpha_{k}$ are given by

$$
\alpha_{k}=\frac{\left\langle u, e_{k}\right\rangle_{2}}{\left\|e_{k}\right\|_{2}^{2}}=\frac{\int_{\Omega} u e_{k} d x}{\int_{\Omega} e_{k}^{2} d x} .
$$

Denote by $\lambda_{u}^{1}$ the least eigenvalue whose associated eigenspace is not orthogonal to $u$. That is,

$$
\lambda_{u}^{1}:=\lambda_{k_{1}} \text { where } k_{1}=\min \left\{k: \alpha_{k} \neq 0\right\} .
$$

In other words, $\lambda_{u}^{1}$ is the first eigenvalue such that $u$ has a non-zero component in the corresponding eigenspace. Note that if $r_{1}$ is the multiplicity of $\lambda_{k_{1}}$ then

$$
\lambda_{u}^{1}=\lambda_{k_{1}}=\lambda_{k_{1}+1}=\cdots=\lambda_{k_{1}+r_{1}-1} .
$$

We will denote by $e_{u}^{1}$ the orthogonal projection of $u$ on the $\lambda_{u}^{1}$-eigenspace, that is

$$
e_{u}^{1}:=\alpha_{k_{1}} e_{k_{1}}+\alpha_{k_{1}+1} e_{k_{1}+1}+\cdots+\alpha_{k_{1}+r_{1}-1} e_{k_{1}+r_{1}-1}=\sum_{\lambda_{k}=\lambda_{u}^{1}} \alpha_{k} e_{k} .
$$

Thus, the Fourier expansion of $u$ can be rewritten as

$$
u=\sum_{\lambda_{k} \geqslant \lambda_{u}^{1}} \alpha_{k} e_{k}=e_{u}^{1}+\sum_{\lambda_{k}>\lambda_{u}^{1}} \alpha_{k} e_{k}=e_{u}^{1}+\sum_{k \geqslant k_{1}+r_{1}} \alpha_{k} e_{k} .
$$

Proceeding in this way, denoting by $e_{u}^{j}$ the orthogonal projection of $u$ on the $\lambda_{u}^{j}$-eigenspace which is the $j$ th-eigenspace not orthogonal to $u$, the eigenfunction expansion of $u$ can be written in terms of its non-zero components in the eigenspaces of the Laplacian as

$$
u=\sum_{j=1}^{M} e_{u}^{j}
$$
where either $M$ is a positive integer or, as in most cases, $M=\infty$, and the corresponding sequence of eigenvalues $\left\{\lambda_{u}^{j}\right\}_{j=1}^{\infty}$ is
(strictly) increasing

$$
0<\lambda_{u}^{1}<\lambda_{u}^{2}<\ldots
$$


As is well known from the theory of compact linear operators, if $\sigma$ does not belong to the spectrum of $-\Delta$ we have that $\left(-\Delta_{\sigma}\right)^{-1}: L^{2}(\Omega) \rightarrow L^{2}(\Omega)$ is a continuous, compact and invertible operator. Therefore, whenever $\sigma$ is not an eigenvalue of the Laplacian, we can define a sequence $\left\{\phi_{n}\right\}_{n \in \mathbb{N}} \subset H_{0}^{1}(\Omega)$ by inverse iteration setting

$$
\phi_{0}=u \quad \text { and } \begin{cases}-\Delta_{\sigma} \phi_{n+1}=\phi_{n} & \text { in } \Omega, \\ \phi_{n+1}=0 & \text { on } \partial \Omega .\end{cases}
$$

For each $u \in L^{2}(\Omega)$ and $\sigma>0$ not in the Laplacian spectrum consider the sequence $\left\{\phi_{n}\right\}$ defined by inverse iteration in (8). Since $\phi_{n+1}=\left(-\Delta_{\sigma}\right)^{-1} \phi_{n}$, it follows from (7) that the eigenfunction expansion of $\phi_{n}$ is given by

$$
\phi_{n}=\sum_{j=1}^{M} \frac{1}{\left(\lambda_{u}^{j}-\sigma\right)^{n}} e_{u}^{j} .
$$

Let $\lambda_{u}^{\sigma}$ be the Laplacian eigenvalue appearing in the spectral decomposition of $u$ which is closest to $\sigma$, i.e.,

$$
\left|\lambda_{u}^{\sigma}-\sigma\right|=\min _{j \in \mathbb{N}}\left|\lambda_{u}^{j}-\sigma\right|
$$

Now let $e_{u}^{\sigma}$ denote the projection of $u$ on the $\lambda_{u}^{\sigma}$-eigenspace. Then

$$
\left\langle u, e_{u}^{\sigma}\right\rangle_{2}=\left\langle e_{u}^{\sigma}, e_{u}^{\sigma}\right\rangle_{2}=\left\|e_{u}^{\sigma}\right\|_{2}^{2}
$$

and we can write

$$
\phi_{n}=\frac{1}{\left(\lambda_{u}^{\sigma}-\sigma\right)^{n}} e_{u}^{\sigma}+\sum_{\left|\lambda_{u}^{j}-\sigma\right|>\left|\lambda_{u}^{\sigma}-\sigma\right|}^{M} \frac{1}{\left(\lambda_{u}^{j}-\sigma\right)^{n}} e_{u}^{j}=\frac{1}{\left(\lambda_{u}^{\sigma}-\sigma\right)^{n}}\left(e_{u}^{\sigma}+\sum_{\left|\lambda_{u}^{j}-\sigma\right|>\left|\lambda_{u}^{\sigma}-\sigma\right|}^{M}\left(\frac{\lambda_{u}^{\sigma}-\sigma}{\lambda_{j}-\sigma}\right)^{n} e_{u}^{j}\right),
$$

or

$$
\phi_{n}=\frac{1}{\left(\lambda_{u}^{\sigma}-\sigma\right)^{n}}\left(e_{u}^{\sigma}+\psi_{n}\right)
$$

where

$$
\psi_{n}=\sum_{\left|\lambda_{u}^{j}-\sigma\right|>\left|\lambda_{u}^{\sigma}-\sigma\right|}^{M}\left(\frac{\lambda_{u}^{\sigma}-\sigma}{\lambda_{u}^{j}-\sigma}\right)^{n} e_{u}^{j} .
$$

Throughout this paper, we will denote by $\lambda_{u}^{\tau}$ the Laplacian eigenvalue appearing in the spectral decomposition of $u$ which is second closest to $\sigma$, i.e.,

$$
\left|\lambda_{u}^{\tau}-\sigma\right|=\min _{\substack{j \in \mathbb{N} \\ \lambda_{u}^{j} \neq \lambda_{u}^{\sigma}}}\left|\lambda_{u}^{j}-\sigma\right| .
$$

We will also denote by $u_{n}$ the component of $u$ in the direction of $\frac{\phi_{n}}{\left\|\phi_{n}\right\|_{2}}$, that is:

$$
u_{n}:=\left\langle u, \frac{\phi_{n}}{\left\|\phi_{n}\right\|_{2}}\right\rangle_{2} \frac{\phi_{n}}{\left\|\phi_{n}\right\|_{2}}=\left(\int_{\Omega} u \frac{\phi_{n}}{\left\|\phi_{n}\right\|_{2}} d x\right) \frac{\phi_{n}}{\left\|\phi_{n}\right\|_{2}} .
$$

\section{3. $L^{2}$-convergence of inverse iteration with shift}

In this section we expose the $L^{2}$-approach of the inverse iteration with shift. Firstly let us state some well-known results concerning the Rayleigh quotient $\mathcal{R}: H_{0}^{1}(\Omega) \backslash\{0\} \rightarrow \mathbb{R}$ defined by

$$
\mathcal{R}(v)=\frac{\int_{\Omega}|\nabla v|^{2} d x}{\int_{\Omega} v^{2} d x}
$$

For ease of consultation, we give short proofs of these results.

Proposition 1. A function $u \in H_{0}^{1}(\Omega) \backslash\{0\}$ is a critical point of $\mathcal{R}$ if and only if $u$ is an eigenfunction of the Laplacian with homogeneous Dirichlet boundary condition and $\mathcal{R}(u)$ is the corresponding eigenvalue.

Proof. Given $v \in H_{0}^{1}(\Omega)$, we have

$$
\mathcal{R}^{\prime}(u) v=\frac{2}{\|u\|_{2}^{2}}\left[\langle\nabla u, \nabla v\rangle_{2}-\mathcal{R}(u)\langle u, v\rangle_{2}\right]
$$


Therefore, $\mathcal{R}^{\prime}(u)=0$ if and only if

$$
\int_{\Omega} \nabla u \cdot \nabla v=\mathcal{R}(u) \int_{\Omega} u v
$$

for all $v \in W_{0}^{1,2}(\Omega)$, that is, $u$ is a weak solution of

$$
\begin{cases}-\Delta u=\mathcal{R}(u) u & \text { in } \Omega, \\ u=0 & \text { on } \partial \Omega .\end{cases}
$$

Corollary 2. The Rayleigh quotient gives a quadratically accurate estimate for the Dirichlet Laplacian eigenvalues, that is, if $u$ is an eigenfunction of the Laplacian with homogeneous Dirichlet boundary condition with $\mathcal{R}(u)$ as the corresponding eigenvalue, then

$$
\mathcal{R}(v)-\mathcal{R}(u)=O\left(\|v-u\|^{2}\right)
$$

as $v \rightarrow u$ in $L^{2}(\Omega)$.

Proof. If follows immediately from Taylor's formula, since $\mathcal{R}^{\prime}(u)=0$.

Now we give the main result of this section. Before this, we note from (11) of Section 2 that

$$
\frac{\phi_{n}}{\left\|\phi_{n}\right\|_{2}}= \pm \frac{e_{u}^{\sigma}+\psi_{n}}{\left\|e_{u}^{\sigma}+\psi_{n}\right\|_{2}},
$$

where the sign of the right-hand side will depend on whether the shift $\sigma$ is taken above or below the eigenvalue. With respect to $n$ : if the shift is taken below the eigenvalue, the sign will always be positive, whereas if the shift is chosen above the eigenvalue the sign will be $(-1)^{n}$.

We note from (14) and (16) that

$$
u_{n}=\left\langle u, \frac{e_{u}^{\sigma}+\psi_{n}}{\left\|e_{u}^{\sigma}+\psi_{n}\right\|_{2}}\right\rangle_{2} \frac{e_{u}^{\sigma}+\psi_{n}}{\left\|e_{u}^{\sigma}+\psi_{n}\right\|_{2}}
$$

and also remark that

$$
\mathcal{R}\left(\phi_{n}\right)=\sigma+\frac{\int_{\Omega} \phi_{n} \phi_{n-1} d x}{\int_{\Omega}\left|\phi_{n}\right|^{2} d x} .
$$

This fact follows from an integration by parts after multiplying the equation $-\Delta_{\sigma} \phi_{n}=\phi_{n-1}$ by $\phi_{n}$. From the computational viewpoint, (18) has the advantage of avoiding the computation of the gradient $\nabla \phi_{n}$ as required in (15).

Theorem 3. Let $u \in L^{2}(\Omega)$.

(i) We have

$$
\left\|\psi_{n}\right\|_{2} \leqslant\left|\frac{\lambda_{u}^{\sigma}-\sigma}{\lambda_{u}^{\tau}-\sigma}\right|^{n}\|u\|_{2} .
$$

In particular, $\psi_{n} \rightarrow 0$ in $L^{2}(\Omega)$ with an exponential rate.

(ii) There exists $n_{0} \in \mathbb{N}$ such that

$$
\left\|\frac{e_{u}^{\sigma}+\psi_{n}}{\left\|e_{u}^{\sigma}+\psi_{n}\right\|_{2}}-\frac{e_{u}^{\sigma}}{\left\|e_{u}^{\sigma}\right\|_{2}}\right\|_{2} \leqslant \frac{4}{\left\|e_{u}^{\sigma}\right\|_{2}}\left\|\psi_{n}\right\|_{2}
$$

for all $n \geqslant n_{0}$. In particular,

$$
\frac{e_{u}^{\sigma}+\psi_{n}}{\left\|e_{u}^{\sigma}+\psi_{n}\right\|_{2}} \rightarrow \frac{e_{u}^{\sigma}}{\left\|e_{u}^{\sigma}\right\|_{2}} \text { in } L^{2}(\Omega),
$$

with an exponential rate.

(iii) The following convergences hold:

$$
\begin{aligned}
& \frac{\left\|\phi_{n}\right\|_{2}}{\left\|\phi_{n+1}\right\|_{2}} \rightarrow\left|\lambda_{u}^{\sigma}-\sigma\right|, \\
& u_{n} \rightarrow e_{u}^{\sigma} \text { in } L^{2}(\Omega),
\end{aligned}
$$


where $u_{n}$ is defined by (14), and

$$
\mathcal{R}\left(\phi_{n}\right) \rightarrow \lambda_{u}^{\sigma}
$$

with

$$
\mathcal{R}\left(\phi_{n}\right)-\lambda_{u}^{\sigma}=O\left(\left|\frac{\lambda_{u}^{\sigma}-\sigma}{\lambda_{u}^{\tau}-\sigma}\right|^{2 n}\right) .
$$

Proof. We have from (12) that

$$
\left\|\psi_{n}\right\|_{2}^{2}=\sum_{\left|\lambda_{u}^{j}-\sigma\right|>\left|\lambda_{u}^{\sigma}-\sigma\right|}^{M}\left|\frac{\lambda_{u}^{\sigma}-\sigma}{\lambda_{u}^{j}-\sigma}\right|^{2 n}\left\|e_{u}^{j}\right\|_{2}^{2} \leqslant\left|\frac{\lambda_{u}^{\sigma}-\sigma}{\lambda_{u}^{\tau}-\sigma}\right|^{2 n} \sum_{\left|\lambda_{u}^{j}-\sigma\right|>\left|\lambda_{u}^{\sigma}-\sigma\right|}^{M}\left\|e_{u}^{j}\right\|_{2}^{2} \leqslant\left|\frac{\lambda_{u}^{\sigma}-\sigma}{\lambda_{u}^{\tau}-\sigma}\right|^{2 n}\|u\|_{2}^{2} .
$$

Since

$$
\left|\frac{\lambda_{u}^{\sigma}-\sigma}{\lambda_{u}^{\tau}-\sigma}\right|<1
$$

it follows that $\left\|\psi_{n}\right\|_{2} \rightarrow 0$ as $n \rightarrow \infty$, which proves (i).

Let $n_{0} \in \mathbb{N}$ be such that

$$
\left\|\psi_{n}\right\|_{2} \leqslant \frac{1}{2}\left\|e_{u}^{\sigma}\right\| \text { for all } n \geqslant n_{0}
$$

Thus, if $n \geqslant n_{0}$ it follows that

$$
\frac{1}{2}\left\|e_{u}^{\sigma}\right\|_{2}=\left\|e_{u}^{\sigma}\right\|_{2}-\frac{1}{2}\left\|e_{u}^{\sigma}\right\|_{2} \leqslant\left\|e_{u}^{\sigma}+\psi_{n}\right\|_{2}+\left\|\psi_{n}\right\|_{2}-\left\|\psi_{n}\right\|_{2}=\left\|e_{u}^{\sigma}+\psi_{n}\right\|_{2}
$$

and

$$
\begin{aligned}
\left\|\frac{e_{u}^{\sigma}+\psi_{n}}{\left\|e_{u}^{\sigma}+\psi_{n}\right\|_{2}}-\frac{e_{u}^{\sigma}}{\left\|e_{u}^{\sigma}\right\|_{2}}\right\|_{2} & =\left\|\frac{\left\|e_{u}^{\sigma}\right\|_{2}\left(e_{u}^{\sigma}+\psi_{n}\right)-e_{u}^{\sigma}\left\|e_{u}^{\sigma}+\psi_{n}\right\|_{2}}{\left\|e_{u}^{\sigma}+\psi_{n}\right\|_{2}\left\|e_{u}^{\sigma}\right\|_{2}}\right\|_{2} \leqslant\left\|\frac{e_{u}^{\sigma}\left(\left\|e_{u}^{\sigma}\right\|_{2}-\left\|e_{u}^{\sigma}+\psi_{n}\right\|_{2}\right)+\left\|e_{u}^{\sigma}\right\|_{2} \psi_{n}}{(1 / 2)\left\|e_{u}^{\sigma}\right\|_{2}^{2}}\right\|_{2} \\
& \leqslant 2 \frac{\left\|e_{u}^{\sigma}\right\|_{2}\left\|\psi_{n}\right\|_{2}+\left\|e_{u}^{\sigma}\right\|_{2}\left\|\psi_{n}\right\|_{2}}{\left\|e_{u}^{\sigma}\right\|_{2}^{2}}=\frac{4}{\left\|e_{u}^{\sigma}\right\|_{2}}\left\|\psi_{n}\right\|_{2},
\end{aligned}
$$

which proves (ii).

Since

$$
\frac{\left\|\phi_{n}\right\|_{2}}{\left\|\phi_{n+1}\right\|_{2}}=\left|\lambda_{u}^{\sigma}-\sigma\right| \frac{\left\|e_{u}^{\sigma}+\psi_{n}\right\|_{2}}{\left\|e_{u}^{\sigma}+\psi_{n+1}\right\|_{2}},
$$

(20) follows from (i).

The $L^{2}$-convergence (21) follows from (17), (19) and (10).

In order to prove (22) we firstly notice that

$$
\lim \left\langle\frac{\phi_{n-1}}{\left\|\phi_{n-1}\right\|_{2}}, \frac{\phi_{n}}{\left\|\phi_{n}\right\|_{2}}\right\rangle_{2}=\left\langle\frac{e_{u}^{\sigma}}{\left\|e_{u}^{\sigma}\right\|_{2}}, \frac{e_{u}^{\sigma}}{\left\|e_{u}^{\sigma}\right\|_{2}}\right\rangle_{2} \begin{cases}1 & \text { if } \lambda_{u}^{\sigma} \geqslant \sigma, \\ -1 & \text { if } \lambda_{u}^{\sigma}<\sigma .\end{cases}
$$

In fact, if $\lambda_{u}^{\sigma} \geqslant \sigma$ then

$$
\lim \left\langle\frac{\phi_{n-1}}{\left\|\phi_{n-1}\right\|_{2}}, \frac{\phi_{n}}{\left\|\phi_{n}\right\|_{2}}\right\rangle_{2}=\lim \left\langle\frac{e_{u}^{\sigma}+\psi_{n-1}}{\left\|e_{u}^{\sigma}+\psi_{n-1}\right\|_{2}}, \frac{e_{u}^{\sigma}+\psi_{n}}{\left\|e_{u}^{\sigma}+\psi_{n}\right\|_{2}}\right\rangle_{2}=\left\langle\frac{e_{u}^{\sigma}}{\left\|e_{u}^{\sigma}\right\|_{2}}, \frac{e_{u}^{\sigma}}{\left\|e_{u}^{\sigma}\right\|_{2}}\right\rangle_{2},
$$

while if $\lambda_{u}^{\sigma}<\sigma$ then

$$
\lim \left\langle\frac{\phi_{n-1}}{\left\|\phi_{n-1}\right\|_{2}}, \frac{\phi_{n}}{\left\|\phi_{n}\right\|_{2}}\right\rangle_{2}=\lim \left\langle(-1)^{n-1} \frac{e_{u}^{\sigma}+\psi_{n-1}}{\left\|e_{u}^{\sigma}+\psi_{n-1}\right\|_{2}},(-1)^{n} \frac{e_{u}^{\sigma}+\psi_{n}}{\left\|e_{u}^{\sigma}+\psi_{n}\right\|_{2}}\right\rangle_{2}=-\left\langle\frac{e_{u}^{\sigma}}{\left\|e_{u}^{\sigma}\right\|_{2}}, \frac{e_{u}^{\sigma}}{\left\|e_{u}^{\sigma}\right\|_{2}}\right\rangle_{2} .
$$

Thus, it follows from (18) that

$$
\lim \mathcal{R}\left(\phi_{n}\right)=\left(\sigma+\left|\lambda_{u}^{\sigma}-\sigma\right|\right)\left\{\begin{array}{ll}
1 & \text { if } \lambda_{u}^{\sigma} \geqslant \sigma, \\
-1 & \text { if } \lambda_{u}^{\sigma}<\sigma .
\end{array}=\lambda_{u}^{\sigma} .\right.
$$


The convergence order (23) follows from (21) and Corollary 2, since

$$
\mathcal{R}\left(\phi_{n}\right)-\lambda_{u}^{\sigma}=\mathcal{R}\left(u_{n}\right)-\mathcal{R}\left(e_{u}^{\sigma}\right)=O\left(\left\|u_{n}-e_{u}^{\sigma}\right\|_{2}^{2}\right)=O\left(\left|\frac{\lambda_{u}^{\sigma}-\sigma}{\lambda_{u}^{\tau}-\sigma}\right|^{2 n}\right) .
$$

\section{Uniform convergence of inverse iteration with shift}

We begin this section by stating a $L^{\infty}$-estimate for an eigenfunction of the Laplacian in terms of its $L^{2}$-norm. In the following, we denote by $|\Omega|$ the Lebesgue measure of $\Omega$.

Lemma 4. Let $e \in H_{0}^{1}(\Omega)$ be an eigenfunction of $-\Delta$ corresponding to the eigenvalue $\lambda$. Then

$$
\|e\|_{\infty} \leqslant 4^{N}|\Omega|^{1 / 2} \lambda^{N / 2}\|e\|_{2} .
$$

Proof. It is shown in [13], without any smoothness assumption on $\partial \Omega$, that if $e$ is an eigenfunction corresponding to a variational eigenvalue $\lambda$ of the homogeneous Dirichlet problem for the $p$-Laplacian then

$$
\|e\|_{\infty} \leqslant 4^{N} \lambda^{N / p}\|e\|_{L^{1}(\Omega)} .
$$

Choosing $p=2$, (24) follows from Hölder's inequality.

Estimates for eigenfunctions of the Laplacian with the exponent $N / 2$ in the eigenvalue replaced by $N / 4$ can be found in [14-17]. See also [18, Remark 5.21] for more references.

The following result refers to the nondecreasing sequence (5) of eigenvalues of the Laplacian.

Lemma 5. If $k>N / 2$, then

$$
\sum_{j=1}^{\infty} \frac{1}{\lambda_{j}^{k}} \leqslant \frac{N C^{k}}{2 k-N}<\infty
$$

where $C$ is a positive constant which depends only on $N$ and $|\Omega|$.

Proof. It is well known (see $[19,20])$ that

$$
\lambda_{j} \geqslant \frac{1}{C} j^{2 / N}
$$

where

$$
C=\frac{N+2}{N} \frac{\left(\omega_{N}|\Omega|\right)^{2 / N}}{4 \pi^{2}}
$$

and $\omega_{N}$ is the volume of the $N$-dimensional unit ball. Hence, if $j>N / 2$ we have

$$
\sum_{j=1}^{\infty} \lambda_{j}^{-k} \leqslant C^{k} \sum_{j=1}^{\infty} j^{-2 k / N}<C^{k} \int_{1}^{\infty} s^{-2 k / N} d s=\frac{N C^{k}}{2 k-N} .
$$

In the next lemma we show that the convergence of a series formed by the eigenvalues $\lambda_{u}^{j}$ which appear in the spectral decomposition of a function $u \in L^{2}(\Omega)$ follows from the convergence of a series formed by all eigenvalues of the Laplacian.

Lemma 6. Let $k$ be chosen so that the series $\sum_{j=1}^{\infty} \frac{1}{\lambda_{j}^{k}}$ is convergent. Then the series

$$
\sum_{j=1}^{M} \frac{\left(\lambda_{u}^{j}\right)^{N / 2}}{\left|\lambda_{u}^{j}-\sigma\right|^{N / 2+k+1}}
$$

is also convergent.

Proof. Assume that the expansion of $u$ is not finite, i.e., $M=\infty$ (otherwise the result is trivial). Since

$$
\sum_{j=1}^{\infty} \frac{1}{\left(\lambda_{u}^{j}\right)^{k}} \leqslant \sum_{j=1}^{\infty} \frac{1}{\lambda_{j}^{k}}
$$


it suffices to show that

$$
\frac{\left(\lambda_{u}^{j}\right)^{N / 2}}{\left|\lambda_{u}^{j}-\sigma\right|^{N / 2+k+1}} \leqslant \frac{1}{\left(\lambda_{u}^{j}\right)^{k}}
$$

for all sufficiently large $j$.

As $\lambda_{u}^{j} \rightarrow \infty$, there exists $j_{0}$ such that $\left|\lambda_{u}^{j}-\sigma\right|=\lambda_{u}^{j}-\sigma$ for all $j \geqslant j_{0}$. Thus, if $j$ is sufficiently large, we can write

$$
\frac{\left(\lambda_{u}^{j}\right)^{N / 2+k}}{\left|\lambda_{u}^{j}-\sigma\right|^{N / 2+k}}=\frac{\left(\lambda_{u}^{j}\right)^{N / 2+k}}{\left(\lambda_{u}^{j}-\sigma\right)^{N / 2+k}}<\lambda_{u}^{j}-\sigma=\left|\lambda_{u}^{j}-\sigma\right|,
$$

whence (27) follows.

In order to prove the uniform convergence of the inverse iteration sequence $\left\{\phi_{n}\right\}_{n \in \mathbb{N}}$, we return to (11) and write

$$
\frac{\phi_{n}}{\left\|\phi_{n}\right\|_{\infty}}= \pm \frac{e_{u}^{\sigma}+\psi_{n}}{\left\|e_{u}^{\sigma}+\psi_{n}\right\|_{\infty}} \text {. }
$$

As in (16), the sign of the right-hand side will depend on whether the shift is taken above or below the eigenvalue and on $n$.

Lemma 7. The inequality

$$
\left\|\psi_{n}\right\|_{\infty} \leqslant K\left|\frac{\lambda_{u}^{\sigma}-\sigma}{\lambda_{u}^{\tau}-\sigma}\right|^{n-\theta}
$$

holds for all sufficiently large $n$, for some $\theta>0$ and a positive constant $K=K\left(u, \Omega,\left|\lambda_{u}^{\sigma}-\sigma\right|\right)$. In particular, $\psi_{n} \rightarrow 0$ uniformly in $\bar{\Omega}$ with an exponential rate.

Proof. From (12) and Lemma 4 we obtain

$$
\left|\psi_{n}\right| \leqslant \sum_{\left|\lambda_{u}^{j}-\sigma\right|>\left|\lambda_{u}^{\sigma}-\sigma\right|}^{M}\left|\frac{\lambda_{u}^{\sigma}-\sigma}{\lambda_{u}^{j}-\sigma}\right|^{n}\left|e_{u}^{j}\right| \leqslant 4^{N}|\Omega|^{1 / 2}\|u\|_{2} \sum_{\left|j_{u}^{j}-\sigma\right|>\left|\lambda_{u}^{\sigma}-\sigma\right|}^{M}\left|\frac{\lambda_{u}^{\sigma}-\sigma}{\lambda_{u}^{j}-\sigma}\right|^{n}\left(\lambda_{u}^{j}\right)^{N / 2} .
$$

But, taking $\theta=N / 2+k+1$, we have

$$
\begin{aligned}
\sum_{\left|\lambda_{u}^{j}-\sigma\right|>\left|\lambda_{u}^{\sigma}-\sigma\right|}^{M}\left|\frac{\lambda_{u}^{\sigma}-\sigma}{\lambda_{u}^{j}-\sigma}\right|^{n}\left(\lambda_{u}^{j}\right)^{N / 2} & =\left|\lambda_{u}^{\sigma}-\sigma\right|^{\theta} \sum_{\left|\lambda_{u}^{j}-\sigma\right|>\left|\lambda_{u}^{\sigma}-\sigma\right|}^{M}\left|\frac{\lambda_{u}^{\sigma}-\sigma}{\lambda_{u}^{j}-\sigma}\right|^{n-\theta} \frac{\left(\lambda_{u}^{j}\right)^{N / 2}}{\left|\lambda_{u}^{j}-\sigma\right|^{\theta}} \leqslant\left|\lambda_{u}^{\sigma}-\sigma\right|^{\theta}\left|\frac{\lambda_{u}^{\sigma}-\sigma}{\lambda_{u}^{\tau}-\sigma}\right|^{n-\theta} \sum_{\left|\lambda_{u}^{j}-\sigma\right|>\left|\lambda_{u}^{\sigma}-\sigma\right|}^{M} \frac{\left(\lambda_{u}^{j}\right)^{N / 2}}{\left|\lambda_{u}^{j}-\sigma\right|^{\theta}} \\
& \leqslant\left|\frac{\lambda_{u}^{\sigma}-\sigma}{\lambda_{u}^{\tau}-\sigma}\right|^{n-\theta}\left|\lambda_{u}^{\sigma}-\sigma\right|^{\theta} \sum_{j=1}^{M} \frac{\left(\lambda_{u}^{j}\right)^{N / 2}}{\left|\lambda_{u}^{j}-\sigma\right|^{\theta}}
\end{aligned}
$$

and by Lemma 6 the last series converges. Thus, (29) follows if we take

$$
K=4^{N}|\Omega|^{1 / 2}\|u\|_{2}\left|\lambda_{u}^{\sigma}-\sigma\right|^{\theta} \sum_{j=1}^{M} \frac{\left(\lambda_{u}^{j}\right)^{N / 2}}{\left|\lambda_{u}^{j}-\sigma\right|^{\theta}} .
$$

Theorem 8. Let $u \in L^{2}(\Omega)$. Then

(i) There exists $n_{0} \in \mathbb{N}$ such that

$$
\left\|\frac{e_{u}^{\sigma}+\psi_{n}}{\left\|e_{u}^{\sigma}+\psi_{n}\right\|_{\infty}}-\frac{e_{u}^{\sigma}}{\left\|e_{u}^{\sigma}\right\|_{\infty}}\right\|_{\infty} \leqslant \frac{4}{\left\|e_{u}^{\sigma}\right\|_{\infty}}\left\|\psi_{n}\right\|_{\infty}
$$

for all $n \geqslant n_{0}$. In particular, 
$\frac{e_{u}^{\sigma}+\psi_{n}}{\left\|e_{u}^{\sigma}+\psi_{n}\right\|_{\infty}} \rightarrow \frac{e_{u}^{\sigma}}{\left\|e_{u}^{\sigma}\right\|_{\infty}}$ uniformly in $\Omega$

with an exponential rate.

(ii) The following convergences hold

$$
\frac{\left\|\phi_{n}\right\|_{\infty}}{\left\|\phi_{n+1}\right\|_{\infty}} \rightarrow\left|\lambda_{u}^{\sigma}-\sigma\right|
$$

and

$u_{n} \rightarrow e_{u}^{\sigma} \quad$ uniformly in $\Omega$.

(iii) If $\mathcal{K} \subset\left\{x: e_{u}^{\sigma}(x) \neq 0\right\}$ is compact, then $\frac{\phi_{n}}{\phi_{n+1}} \rightarrow \lambda_{u}^{\sigma}-\sigma$ uniformly and with an exponential rate.

Proof. Let $n_{0}$ be such that $\left\|\psi_{n}\right\|_{\infty} \leqslant \frac{1}{2}\left\|e_{u}^{\sigma}\right\|_{\infty}$ for all $n \geqslant n_{0}$. Then, as in the proof of (ii) of Theorem 3 , we have for all $n \geqslant n_{0}$ that

$$
\frac{1}{2}\left\|e_{u}^{\sigma}\right\|_{\infty} \leqslant\left\|e_{u}^{\sigma}+\psi_{n}\right\|_{\infty}
$$

and

$$
\left\|\frac{e_{u}^{\sigma}+\psi_{n}}{\left\|e_{u}^{\sigma}+\psi_{n}\right\|_{\infty}}-\frac{e_{u}^{\sigma}}{\left\|e_{u}^{\sigma}\right\|_{\infty}}\right\|_{\infty} \leqslant \frac{4}{\left\|e_{u}^{\sigma}\right\|_{\infty}}\left\|\psi_{n}\right\|_{\infty} .
$$

The remaining of (i) follows from Lemma 7.

In order to prove (31), write

$$
u_{n}=\left(\frac{\left\|\phi_{n}\right\|_{\infty}}{\left\|\phi_{n}\right\|_{2}}\right)^{2} \frac{\phi_{n}}{\left\|\phi_{n}\right\|_{\infty}} \int_{\Omega} u \frac{\phi_{n}}{\left\|\phi_{n}\right\|_{\infty}} d x
$$

Since

$$
\lim \frac{\left\|\phi_{n}\right\|_{\infty}}{\left\|\phi_{n}\right\|_{2}}=\lim \frac{\left\|e_{u}^{\sigma}+\psi_{n}\right\|_{\infty}}{\left\|e_{u}^{\sigma}+\psi_{n}\right\|_{2}}=\frac{\left\|e_{u}^{\sigma}\right\|_{\infty}}{\left\|e_{u}^{\sigma}\right\|_{2}}
$$

and, from (i),

$$
\frac{\phi_{n}}{\left\|\phi_{n}\right\|_{\infty}} \int_{\Omega} u \frac{\phi_{n}}{\left\|\phi_{n}\right\|_{\infty}} d x \rightarrow \frac{e_{u}^{\sigma}}{\left\|e_{u}^{\sigma}\right\|_{\infty}} \int_{\Omega} u \frac{e_{u}^{\sigma}}{\left\|e_{u}^{\sigma}\right\|_{\infty}} d x
$$

uniformly in $\Omega$, it follows from (10) that

$$
u_{n} \rightarrow\left(\frac{\left\|e_{u}^{\sigma}\right\|_{\infty}}{\left\|e_{u}^{\sigma}\right\|_{2}}\right)^{2} \frac{e_{u}^{\sigma}}{\left\|e_{u}^{\sigma}\right\|_{\infty}} \int_{\Omega} u \frac{e_{u}^{\sigma}}{\left\|e_{u}^{\sigma}\right\|_{\infty}} d x=\frac{e_{u}^{\sigma}}{\left\|e_{u}^{\sigma}\right\|_{2}^{2}} \int_{\Omega} u e_{u}^{\sigma} d x=\frac{e_{u}^{\sigma}}{\left\|e_{u}^{\sigma}\right\|_{2}^{2}}\left\langle u, e_{u}^{\sigma}\right\rangle=e_{u}^{\sigma}
$$

Since from (11) we have

$$
\frac{\left\|\phi_{n}\right\|_{\infty}}{\left\|\phi_{n+1}\right\|_{\infty}}=\left|\lambda_{u}^{\sigma}-\sigma\right| \frac{\left\|e_{u}^{\sigma}+\psi_{n}\right\|_{\infty}}{\left\|e_{u}^{\sigma}+\psi_{n+1}\right\|_{\infty}}
$$

and thus (30) also follows from Lemma 7.

Now, let $\mathcal{K} \subset \subset$ suppe $_{u}^{\sigma}$ be compact so that

$$
m:=\min _{\mathcal{K}}\left|e_{u}^{\sigma}\right|>0
$$

and fix $n_{0} \in \mathbb{N}$ such that

$$
\left\|\psi_{n}\right\|_{\infty}<\frac{m}{2} \text { for all } n \geqslant n_{0} .
$$

Thus if $n \geqslant n_{0}$ we have on $\mathcal{K}$

$$
\left|e_{u}^{\sigma}+\psi_{n}\right| \geqslant\left|e_{u}^{\sigma}\right|-\left|\psi_{n}\right| \geqslant m-\left\|\psi_{n}\right\|_{\infty}>\frac{m}{2} .
$$

Therefore, the quotient 


$$
\frac{\phi_{n}}{\phi_{n+1}}=\left(\lambda_{u}^{\sigma}-\sigma\right) \frac{e_{u}^{\sigma}+\psi_{n}}{e_{u}^{\sigma}+\psi_{n+1}}
$$

makes sense on $\mathcal{K}$ for all sufficiently large $n$ and again (iii) follows from Lemma 7 since

$$
\left|\frac{\phi_{n}}{\phi_{n+1}}-\left(\lambda_{u}^{\sigma}-\sigma\right)\right|=\left|\lambda_{u}^{\sigma}-\sigma\right|\left|\frac{e_{u}^{\sigma}+\psi_{n}}{e_{u}^{\sigma}+\psi_{n+1}}-1\right|=\left|\lambda_{u}^{\sigma}-\sigma\right|\left|\frac{\psi_{n}-\psi_{n+1}}{e_{u}^{\sigma}+\psi_{n+1}}\right| \leqslant \frac{2\left|\lambda_{u}^{\sigma}-\sigma\right|}{m}\left|\psi_{n}-\psi_{n+1}\right| .
$$

\section{Normalization at each step}

In order to avoid numerical problems, such as overflow or underflow, it is usual to normalize the right-hand-side function in each inverse iteration. Although this procedure changes the sequence of iterates it maintains convergences.

In fact, let $v_{n}$ be defined by

$$
v_{0}=\frac{u}{\|u\|} \quad \text { and } \begin{cases}-\Delta_{\sigma} v_{n+1}=\frac{v_{n}}{\left\|v_{n}\right\|} & \text { in } \Omega \\ v_{n+1}=0 & \text { on } \partial \Omega\end{cases}
$$

where $\|\cdot\|$ may denote the $L^{2}$-norm or the $L^{\infty}$-norm. Then, since $\sigma$ is not an eigenvalue of $-\Delta$ it is easy to verify that

$$
\phi_{n+1}=\left\|\phi_{n}\right\| v_{n+1} .
$$

Hence, for example, if one uses $\|\cdot\|=\|\cdot\|_{2}$ then

$$
\left\langle u, \frac{v_{n}}{\left\|v_{n}\right\|_{2}}\right\rangle_{2} \frac{v_{n}}{\left\|v_{n}\right\|_{2}}=u_{n} \rightarrow e_{u}^{\sigma}
$$

both uniformly and in $L^{2}$. Note that this sequence is exactly the sequence $\left\{u_{n}\right\}$ defined by (14) and rewritten in terms of the sequence $\left\{v_{n}\right\}$.

Moreover, in view of (33) and (15) one also has

$$
\mathcal{R}\left(v_{n}\right)=\mathcal{R}\left(\phi_{n}\right) \rightarrow \lambda_{u}^{\sigma} \text {. }
$$

If $\|\cdot\|=\|\cdot\|_{\infty}$ then one has the following uniform convergence in each compact $\mathcal{K} \subset\left\{x: e_{u}^{\sigma}(x) \neq 0\right\}$ :

$$
\frac{v_{n}}{v_{n+1}} \rightarrow \frac{\lambda_{u}^{\sigma}-\sigma}{\left|\lambda_{u}^{\sigma}-\sigma\right|}= \begin{cases}1 & \text { if } \lambda_{u}^{\sigma}>\sigma \\ -1 & \text { if } \lambda_{u}^{\sigma}<\sigma .\end{cases}
$$

\section{Numerical tests}

In this section we present some numerical tests on the unit interval, unit disk and unit square. The inverse iteration with shift was implemented starting from the unit constant function $u \equiv 1$ on these domains. Eigenvalue approximations were computed by running our Algorithm 1 and taking (in the line 9 ) the following sequences considered in this paper:

$$
\mu_{n}:=\frac{\phi_{n}}{\phi_{n+1}}\left(x_{0}\right)+\sigma, \quad \gamma_{n}:=\frac{\left\|\phi_{n}\right\|_{2}}{\left\|\phi_{n+1}\right\|_{2}}+\sigma
$$

and

$$
\mathcal{R}\left(\phi_{n}\right)=\sigma+\frac{\left\langle\phi_{n}, \phi_{n-1}\right\rangle_{2}}{\left\|\phi_{n}\right\|_{2}^{2}} .
$$

This last sequence is an alternative form of the Rayleigh quotient evaluated at $\phi_{n}$ according to (18). As previously remarked, the numerical advantage of writing the Rayleigh quotient in this form is that one need not compute the gradient $\nabla \phi_{n}$.

Taking into account (35) we also compute eigenvalue approximations using the sequence $\mathcal{R}\left(v_{n}\right)$ where $v_{n}$ is defined by (32). Namely, we use the following alternative expression for this sequence:

$$
\mathcal{R}\left(v_{n}\right)=\sigma+\frac{\left\langle v_{n}, v_{n-1}\right\rangle_{2}}{\left\|v_{n}\right\|_{2}^{2}} .
$$

Notwithstanding the (theoretical) equality $\mathcal{R}\left(v_{n}\right)=\mathcal{R}\left(\phi_{n}\right)$, our tests indicate some remarkable numerical differences between them.

The non-normalization of the function $\phi_{n}$ on each step in Algorithm 1 tends to attribute to it smaller values at each iteration. Thus, as a numerical phenomenon, the quotient $\phi_{n} / \phi_{n+1}$ tends to assume the value 1 , what makes the sequences $\mu_{n}, \gamma_{n}$ and $\mathcal{R}\left(\phi_{n}\right)$ converge to $\sigma+1$.

This phenomenon may restrict the use of these sequences. However, handled with care by controlling the number of iterations and points in the grid, they can provide good approximations to the eigenvalues, as our numerical tests indicate. 
Table 1

Exact and approximated eigenvalues on the unit interval $[0,1]$ obtained from the inverse iteration with shift starting from the unit function. The shift $\sigma_{k}:=\lambda_{k}-0.1$ and a grid containing 101 nodes were used.

\begin{tabular}{|c|c|c|c|c|c|}
\hline$k$ & $\lambda_{k}$ & $\mu_{10}$ & $\gamma_{10}$ & $\mathcal{R}\left(\phi_{10}\right)$ & $\mathcal{R}\left(v_{10}\right)$ \\
\hline 1 & 9.8696 & 9.8688 & 9.8688 & 9.8688 & 9.8688 \\
\hline 2 & 39.4784 & 39.4654 & 39.4654 & 39.4654 & 9.8691 \\
\hline 3 & 88.8264 & 88.7607 & 88.7607 & 88.7607 & 88.7607 \\
\hline 4 & 157.914 & 157.706 & 157.921 & 157.706 & 89.1623 \\
\hline 5 & 246.740 & 246.233 & 247.047 & 246.233 & 246.233 \\
\hline 6 & 355.306 & 354.255 & 356.157 & 356.206 & 252.178 \\
\hline 7 & 483.611 & 481.665 & 485.356 & 481.665 & 481.665 \\
\hline 8 & 631.655 & 628.335 & 634.773 & 632.555 & 514.785 \\
\hline
\end{tabular}
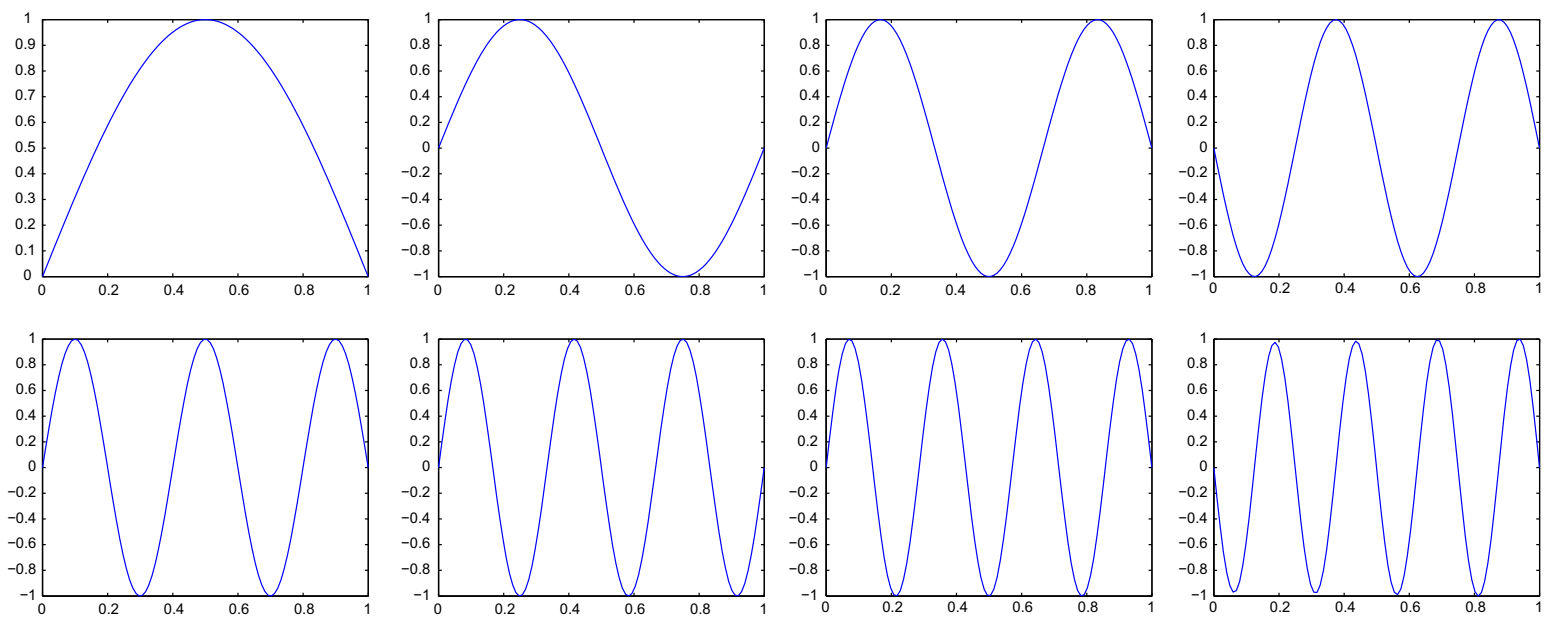

Fig. 1. First eight approximated eigenfunctions of the Laplacian on $[0,1]$ obtained from the inverse iteration with shift starting from the unit function

Table 2

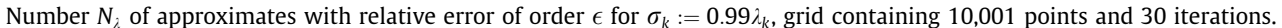

\begin{tabular}{lllllll}
\hline$\epsilon$ & $10^{-3}$ & $10^{-4}$ & $10^{-5}$ & $10^{-6}$ & $10^{-7}$ & $\leqslant 10^{-8}$ \\
\hline$N_{\lambda}$ & 292 & 1004 & 156 & 39 & 6 \\
\hline
\end{tabular}

On the other hand, the sequence $\mathcal{R}\left(v_{n}\right)$ seems to be more robust, since we did not observe this phenomenon when using it. Another indication of its numerical stability is its tendency to better capture the correct eigenvalues (i. e. those belonging to the spectrum of the starting function), than the other sequences.

The graphs of the eigenfunction approximations were constructed by using the sequence $u_{n}$ in (34). In Algorithm 2, these approximations can be viewed as the combination of lines 9 and 10 with $\phi_{n+1}^{k}$ replaced by $v_{n+1}^{k}$ (normalizing on each step).

To show the efficiency of inverse iteration with shift we used neither the most efficient available method for solving the underlying differential equation nor a fine grid, but one of the most basic methods, finite differences, and a relatively coarse grid. Integrals were computed via the Simpson composite method.

\subsection{Eigenvalues and eigenfunctions for the unit interval $[0,1]$}

In this case, (8) becomes the following boundary value problem

$$
\left\{\begin{array}{l}
-\phi_{n+1}^{\prime \prime}-\sigma \phi_{n+1}=\phi_{n} \\
\phi_{n+1}(0)=\phi_{n+1}(1)=0
\end{array}\right.
$$

One can verify that $\lambda_{k}=k^{2} \pi^{2}$ and that the function $u \equiv 1$ does not have components corresponding to $\lambda_{k}$ for $k$ even.

We present in Table 1 exact and approximated eigenvalues of the Laplacian on the unit interval. The shift was set to the corresponding exact eigenvalue minus 0.1 (that is, $\sigma_{k}:=\lambda_{k}-0.1$ ), and a grid of only 101 nodes was used. As shown in the last column, the sequence $\mathcal{R}\left(v_{n}\right)$ seems to capture only the eigenvalues $\lambda_{u}^{\sigma}$ that appear on the spectral decomposition of the 
Table 3

Number $N_{\lambda}$ of approximates with relative error of order $\epsilon$ for $\sigma_{k}:=0.5\left(\lambda_{k+1}+\lambda_{k}\right)$, grid containing 10,001 points and 30 iterations.

\begin{tabular}{llllll}
\hline$\epsilon$ & $10^{-4}$ & $10^{-5}$ & $10^{-6}$ & $10^{-7}$ \\
\hline$N_{\lambda}$ & 1 & 66 & 25 & 4 \\
\hline
\end{tabular}

Table 4

Numbers $N_{\sigma}$ of shifts and $N_{\lambda}$ of approximates with relative errors of order $\epsilon$. Here $\sigma$ was randomly chosen on the interval $\left(0, \lambda_{50}\right)$. A grid containing 10,001 points and 30 iterations were used.

\begin{tabular}{llllll}
\hline$\epsilon$ & $\geqslant 10^{-2}$ & $10^{-3}$ & $10^{-4}$ & $10^{-5}$ & $10^{-6}$ \\
\hline$N_{\sigma}$ & 38 & 60 & 2 & 0 & 0 \\
$N_{\lambda}$ & 0 & 1 & 2 & 80 & $10^{-7}$ \\
\hline
\end{tabular}

function $u \equiv 1$. In fact, we note that for even values of $k$ the shift is closer to $\lambda_{k}$ than $\lambda_{k-1}$ and even so the corresponding sequence $\mathcal{R}\left(v_{n}\right)$ converges to $\lambda_{k-1}$ which is the correct eigenvalue.

In Fig. 1 we present the graphs of the first eight approximated eigenfunctions.

Table 1 also exemplifies the numerical convergence to $\sigma+1$ of the non-normalized sequence $\mathcal{R}\left(\phi_{n}\right)$, which happened to the approximations of $\lambda_{6}$ and $\lambda_{8}$. For the first one, for example, the closest approximation achieved is $\mathcal{R}\left(\phi_{4}\right)=280.278$. For $n>4$ the quotient collapses to one and the result is spurious. In order to compute a correct approximation of this eigenvalue using this sequence, a finer grid should be used.

In Table 2 we show the result of calculating the first 1,500 eigenvalues of the unit interval using the normalized sequence $\mathcal{R}\left(v_{n}\right)$ with 30 iterations and a grid containing 10,001 nodes. The relative error between the computed eigenvalue $\mathcal{R}\left(v_{n}\right)$ and the exact eigenvalue $\lambda$ is defined by

$$
\epsilon\left(\mathcal{R}\left(v_{n}\right), \lambda\right)=\left|\frac{\mathcal{R}\left(v_{n}\right)-\lambda}{\lambda}\right| .
$$

The shift used to make Table 2 is $\sigma_{k}:=0.99 \lambda_{k}$, which has an initial relative error of $1 \%$. Such error is huge for great eigenvalues and the interval $\left(\sigma_{k}, \lambda_{k}\right)$ may contain many other eigenvalues. Thus, it is likely to happen that this shift makes the sequence converge to an eigenvalue $\lambda_{r}$ different from $\lambda_{k}$. With this in mind, we considered that $\mathcal{R}\left(v_{30}\right)$ correctly approximated an eigenvalue $\lambda_{r}$ if $\left|\lambda_{r}-\mathcal{R}\left(v_{30}\right)\right|=\min _{s}\left|\lambda_{s}-\mathcal{R}\left(v_{30}\right)\right|$, and that $\mathcal{R}\left(v_{30}\right)$ converged to $\lambda_{r}$ if their relative error is less than $10^{-3}$

Table 2 reveals that among the 1,500 shifts used, all of them approximated an eigenvalue with a relative error of order of magnitude $10^{-3}$, and that 1,208 converged with a relative error less than $10^{-3}$.

Among the 1,208 converged approximations, 547 refer to eigenvalues $\lambda_{k}$ with $k$ even. This shows that the sequence $\mathcal{R}\left(v_{n}\right)$ can converge to an eigenvalue that does not belong to the spectrum of the unit function.

In order to better understand convergence of shifts with large initial relative error, we used a grid of 10,001 nodes and 30 iterations of the sequence $\mathcal{R}\left(v_{n}\right)$ with shifts $\sigma_{k}:=0.5\left(\lambda_{k+1}+\lambda_{k}\right)$, where $k$ runs from 1 to 100 . The result is presented in Table 3. As we can see, despite the shift being located exactly between the eigenvalues, only one did not converge to an eigenvalue.

Another numerical experiment using shifts with large initial error was done with randomly chosen shifts. We generated 100 random numbers (shifts) on the interval $\left(0, \lambda_{50}\right)$ and used a grid of 10,001 nodes and 30 iterations. Table 4 shows the initial relative errors of the shifts, as well as the errors after 30 iterations of sequence $\mathcal{R}\left(v_{n}\right)$. As we can see, only one of these shifts did not converge to an eigenvalue, and most of them converged with a relative error of order $10^{-5}$.

\subsection{Radial eigenvalues and eigenfunctions for the unit disk}

We computed only the radial eigenfunctions for the unit disk $\Omega=\left\{x \in \mathbb{R}^{2}:|x| \leqslant 1\right\}$. In this case $\phi_{n}=\phi_{n}(r)$ where $r=|x|$, and (8) becomes the Sturm-Liouville problem type

$$
\left\{\begin{array}{l}
-\frac{\left(r \phi_{n+1}^{\prime}\right)^{\prime}}{r}-\sigma \phi_{n+1}=\phi_{n}, 0<r<1, \\
\phi_{n+1}^{\prime}(0)=0=\phi_{n+1}(1) .
\end{array}\right.
$$

Note that the function $u \equiv 1$ has components in all radial eigenspaces. In fact, if $e=e(r)$ denotes a radial eigenfunction corresponding to an eigenvalue $\lambda>0$ in $\Omega$ then

$$
\left\{\begin{array}{l}
\Delta_{r} e=\frac{\left(r e^{\prime}\right)^{\prime}}{r}=-\lambda e, 0<r<1 \\
e^{\prime}(0)=0=e(1)
\end{array}\right.
$$

Therefore, 
Table 5

The first eight Laplacian radial eigenvalues on the unit disk obtained from the inverse iteration with shift starting from the unit function.

\begin{tabular}{|c|c|c|c|c|c|}
\hline$k$ & $\lambda_{k}$ & $\mu_{10}$ & $\gamma_{10}$ & $\mathcal{R}\left(\phi_{10}\right)$ & $\mathcal{R}\left(v_{10}\right)$ \\
\hline 1 & 5.7831 & 5.7834 & 5.7834 & 5.7392 & 5.7392 \\
\hline 2 & 30.4713 & 30.4698 & 30.4698 & 30.4396 & 30.4396 \\
\hline 3 & 74.887 & 74.865 & 74.865 & 74.847 & 74.847 \\
\hline 4 & 139.040 & 138.942 & 138.942 & 138.942 & 138.942 \\
\hline 5 & 222.932 & 222.646 & 223.018 & 222.674 & 222.674 \\
\hline 6 & 326.563 & 325.901 & 327.025 & 325.968 & 326.563 \\
\hline 7 & 449.934 & 448.611 & 451.057 & 448.729 & 448.729 \\
\hline 8 & 593.043 & 590.663 & 595.223 & 590.836 & 590.836 \\
\hline
\end{tabular}
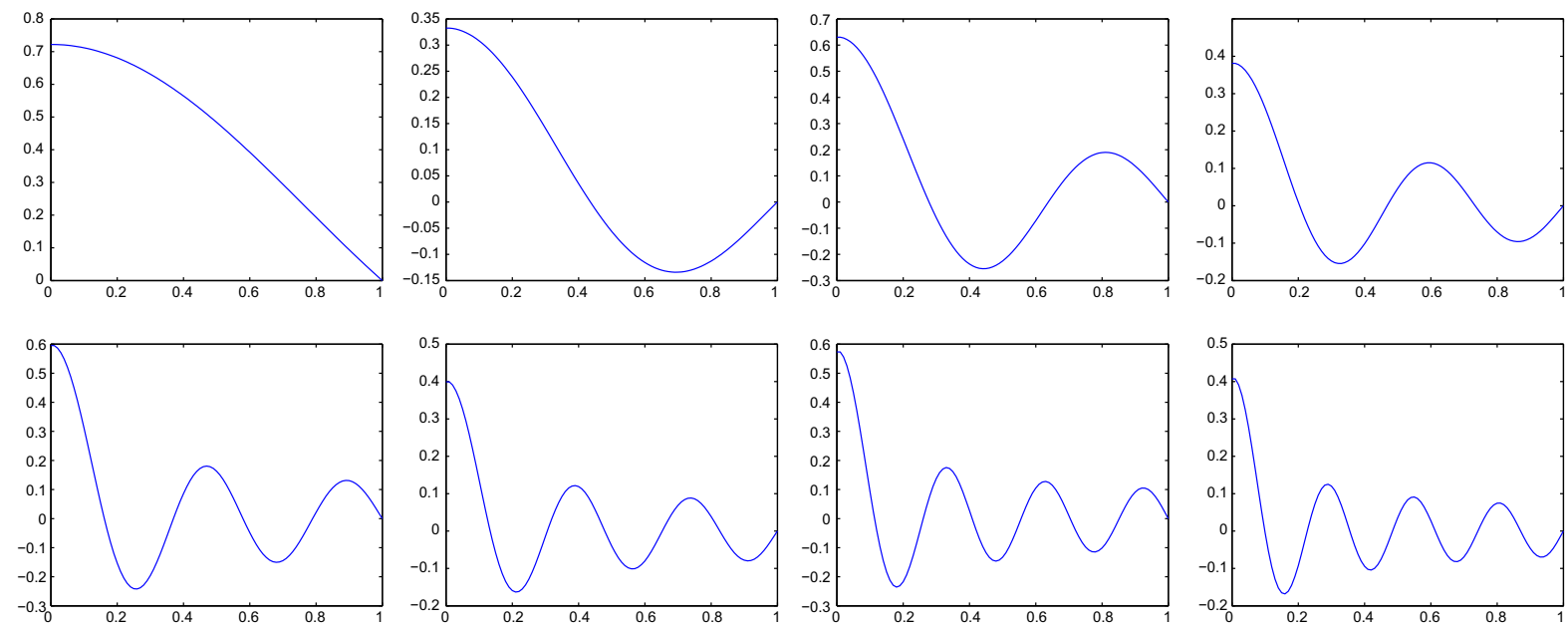

Fig. 2. First eight Laplacian radial eigenfunctions on the unit disk obtained from the inverse iteration with shift.

Table 6

Exact and approximated eigenvalues of the Laplacian on the unit square.

\begin{tabular}{lcccc}
\hline$(n, m)$ & $\lambda_{n, m}$ & $\mu_{10}$ & $\gamma_{10}$ & $\mathcal{R}\left(\phi_{10}\right)$ \\
\hline$(1,1)$ & 19.7392 & 19.7388 & 19.7388 & 19.7388 \\
$(1,2)$ & 49.3480 & 49.3346 & 49.3446 & 49.3446 \\
$(2,2)$ & 78.9568 & 78.9504 & 78.9504 & 78.7388 \\
$(1,3)$ & 98.6960 & 98.6796 & 98.6308 & 19.8395 \\
$(2,3)$ & 128.305 & 128.285 & 128.285 & 98.6732 \\
$(3,3)$ & 177.653 & 177.620 & 177.620 & 98.6796 \\
\hline
\end{tabular}
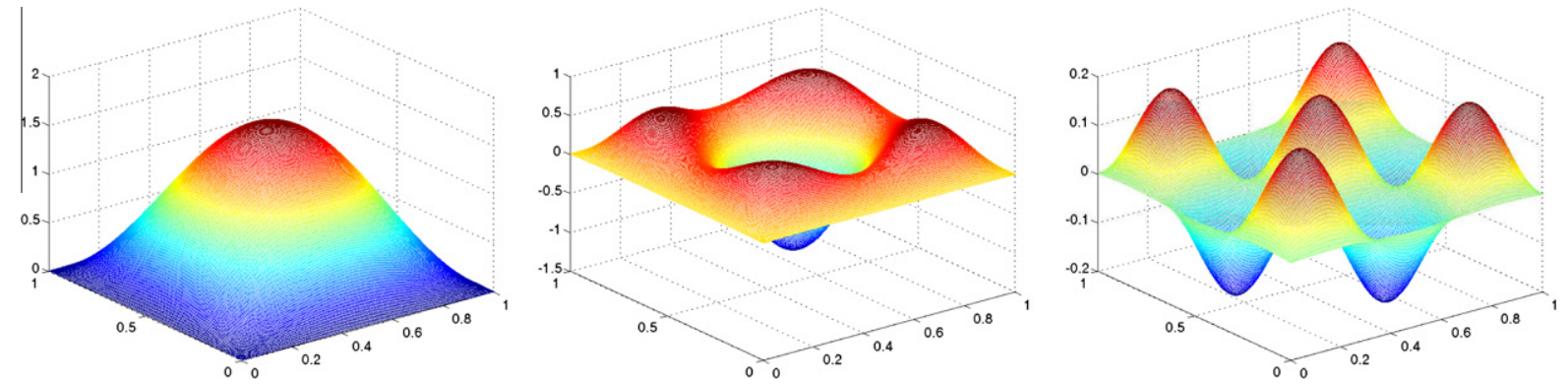

Fig. 3. Graphs of the approximations for the first three projections of the function $u \equiv 1$ on the unit square obtained of the inverse iteration with shift $\sigma_{n, m}:=\lambda_{n, m}-0.1 . e_{u}^{\sigma_{1,1}}$ (left), $e_{u}^{\sigma_{1,3}}$ (center) and $e_{u}^{\sigma_{3,3}}$ (right). 
Table 7

Approximated eigenvalues of the Laplacian on the unit square and relative errors for different grids. The exact eigenvalue is $\lambda_{3,3}=177.6529$.

\begin{tabular}{lll}
\cline { 2 - 3 } Grid & $\mu_{10}$ & $\epsilon\left(\mu_{10}, \lambda_{3,3}\right)$ \\
\hline $100 \times 100$ & 177.5187 & $7.6 \times 10^{-4}$ \\
$200 \times 200$ & 177.6197 & $1.9 \times 10^{-4}$ \\
$300 \times 300$ & 177.6382 & $8.3 \times 10^{-5}$ \\
$400 \times 400$ & 177.6446 & $4.7 \times 10^{-5}$ \\
$500 \times 500$ & 177.6476 & $3.0 \times 10^{-5}$ \\
$1000 \times 1000$ & 177.6516 & $7.4 \times 10^{-6}$ \\
$2000 \times 2000$ & 177.6526 & $1.9 \times 10^{-6}$ \\
\hline
\end{tabular}

$$
\langle u, 1\rangle_{2}=\int_{|x| \leqslant 1} e(|x|) d x=\int_{0}^{1} \int_{|x|=r} e(r) d S_{x} d r=2 \pi \int_{0}^{1} e(r) r d r=-\frac{2 \pi}{\lambda} \int_{0}^{1}\left(r e^{\prime}(r)\right)^{\prime} d r=-\frac{2 \pi}{\lambda} e^{\prime}(1) \neq 0
$$

because of the uniqueness of the initial value problems for the ODE above at $r=1$.

We present in Table 5 the exact and approximated first eight radial eigenvalues for the Laplacian on the unit disk, calculated using the shift $\sigma_{k}:=\lambda_{k}-0.1$ and a grid containing 201 nodes.

The graphs of the first eight approximated radial eigenfunctions of the Laplacian obtained by our Algorithm 2 are displayed in Fig. 2.

\subsection{Unit square}

The eigenvalues of the unit square $\Omega=[0,1] \times[0,1]$ are $\lambda_{n, m}=\left(n^{2}+m^{2}\right) \pi^{2}$ and the corresponding $L^{\infty}$-normalized eigenfunctions are $e_{n, m}=\sin (n \pi x) \sin (m \pi x)$. Hence it is easy to verify that the spectrum of the function $u \equiv 1$ consists precisely of those eigenvalues $\lambda_{n, m}$ for which both $n$ and $m$ are odd and that its first three eigenvalues are $\lambda_{1,1}, \lambda_{1,3}$ and $\lambda_{3,3}$. In Table 6 we present exact and approximated eigenvalues of the Laplacian on this domain. The shift was set $\sigma_{k}:=\lambda_{k}-0.1$ and a grid containing $201 \times 201$ nodes was used. We can see again that the sequence $\mathcal{R}\left(v_{n}\right)$ tends to capture only the eigenvalues $\lambda_{u}^{\sigma}$ that appear on the spectrum of the function $u \equiv 1$, as shown in the last column. Note that the shift $\sigma_{1,2}:=\lambda_{1,2}-0.1$ is closer to $\lambda_{1,2}$ but, however, the corresponding sequence $\mathcal{R}\left(v_{n}\right)$ approaches the correct eigenvalue $\lambda_{1,1}$. The same behavior happens with the shifts $\sigma_{2,2}:=\lambda_{2,2}-0.1$ and $\sigma_{2,3}:=\lambda_{2,3}-0.1$ since they are closer to $\lambda_{2,2}$ and $\lambda_{2,3}$, respectively, but the corresponding sequences $\mathcal{R}\left(v_{n}\right)$ approach to $\lambda_{1,3}$. The graphs of the first three eigenfunctions in the spectrum of the unit function using Algorithm 2 are displayed in Fig. 3.

In Table 7 we used the sequence $\mu_{n}$ to show the effect of refining the grid. The shift was chosen $\sigma_{3,3}:=\lambda_{3,3}-0.1$ and 10 iterations were used. As expected, a finer grid provides a better approximation of the eigenvalue.

\section{Final comments}

In finite linear algebra, the iterative process itself is often used in order to generate increasingly better estimates for the eigenvalue at each iteration, meaning that the approximation obtained at any given iteration is used as the shift in the next iteration. It turns out that instead of using the estimates for the eigenvalue obtained in the process, the Rayleigh quotient of the estimates for the eigenvector obtained at each iteration give much better approximations for the eigenvalue. Indeed, if the eigenvalues of the operator or at least very good estimates of them are known in advance, inverse iteration with shift given by the Rayleigh quotient is the standard method for computing eigenvalues due to its cubic rate of convergence (see [2]). It would be only natural to extend such ideas to the Laplacian, but we were not able to do it. Instead, our (admittedly preliminary) numerical tests, not shown in this paper, did not indicate convergence to the correct eigenvalues. As previously discussed, the Rayleigh quotient may not be a good way to approximate the eigenvalue of high frequency eigenfunctions unless the grid is much further refined, due to high oscillations, and the computational cost of using too fine grids can seriously limit the efficiency of the method. Further investigation is needed. So it remains an open problem to us if inverse iteration with shift given by the Rayleigh quotient is a method that can be successfully applied to the Laplacian.

The method described in this paper uses a modification of the Rayleigh quotient that avoids the computation of gradients. Our numerical tests shown in Section 6 indicate a greater degree of convergence to the correct eigenvalues when this form is used. It seems to us that the non-necessity of calculating the gradients makes our algorithm more numerically stable.

The only reference we could find where the Rayleigh quotient was used in computing the eigenvalues of the Laplacian, and only for polygonal domains, was the work [21]; however the Rayleigh quotient was only indirectly used there, as one component of another algorithm and in a very different way from the direct approach we follow here.

\section{Acknowledgments}

The authors thank the support of FAPEMIG and CNPq-Brazil. 


\section{References}

[1] R.J. Biezuner, G. Ercole, E.M. Martins, Computing the first eigenvalue of the p-Laplacian via the inverse power method, J. Func. Anal. 257 (2009) 243270.

[2] L.N. Trefenthen, D. Bau III, Numerical Linear Algebra, SIAM, 1997.

[3] J.R. Kuttler, V.G. Sigillito, Eigenvalues of the Laplacian in two dimensions, SIAM Rev. 26 (2) (1984) $163-193$.

[4] G.N. Hile, M.H. Protter, Inequalities for eigenvalues of the Laplacian, Indiana Univ. Math. J. 29 (1980) 523-528.

[5] H.C. Yang, Estimates of the difference between consecutive eigenvalues, preprint, 1995 (revision of International Centre for Theoretical Physics preprint IC/91/60, Trieste, Italy, April 1991), revised preprint, from Academica Sinica, 1995.

[6] Q.-M. Cheng, H. Yang, Bounds on eigenvalues of Dirichlet Laplacian, Mathematische Annalen 337 (1) (2007) $159-175$.

[7] K. Uhlenbeck, Eigenfunctions of Laplace operator, Bull. Amer. Math. Soc. 78 (1972) 1073-1076.

[8] K. Uhlenbeck, Generic properties of eigenfunctions, Amer. J. Math. 98 (1976) 1059-1078.

[9] W. Hackbusch, Elliptic Differential Equations: Theory and Numerical Treatment, Springer Series in Computational Mathematics, 18, Springer, 1992.

[10] V. Heuveline, On the computation of a very large number of eigenvalues for selfadjoint elliptic operators by means of multigrid methods, J. Comput. Phys. 184 (2003) 321-337.

[11] H. Weyl, Über die asymptotische verteilung der eigenwerte, Nachr. Konigl. Ges. Wiss. Göttingen (1911) $110-117$.

[12] R. Courant, D. Hilbert, Methods of Mathematical Physics, Wiley Interscience, 1953.

[13] P. Lindqvist, in: On a nonlinear eigenvalue problem, topics in mathematical analysis, Series on Analysis Applications and Computation, vol. 3, World Sci. Publ., 2008, pp. 175-203.

[14] Y.V. Egorov, V.A. Kondrat'ev, Some estimates for eigenfunctions of an elliptic operator, (Russian) Vestnik Moskov. Univ. Ser. I Mat. Mekh 105 (4) (1985) 32-34. English translation: Moscow Univ. Math. Bull. 40 (4) (1985) 49-52.

[15] V.Y. Yakubov, Estimates for elliptic operator eigenfunctions normalized in $\mathrm{L}_{2}$, Dokl. Akad. Nauk SSSR 274 (1) (1984) 35-37. English transl.: Soviet Math. Dokl. 29 (1984) 29-31.

[16] V.Y. Yakubov, Sharp estimates for $\mathrm{L}_{2}$-normalized eigenfunctions of an elliptic operator, Dokl. Ross. Akad. Nauk 331 (3) (1993) 286-287. English transl.: Russian Acad. Sci. Dokl. Math. 48 (1) (1994) 92-94.

[17] V.Y. Yakubov, (Russian) Estimates for the eigenfunctions of elliptic operators with respect to the spectral parameter, Funktsional. Anal. i Prilozhen. 33 (2) (1999) 58-67,96. English transl.: Funct. Anal. Appl. 33 (2) (1999) 128-136.

[18] V.I. Burenkov, P.D. Lamberti, Spectral stability of Dirichlet second order uniformly elliptic operators, J. Differ. Equat. 244 (2008) $1712-1740$.

[19] P. Li, S.-T. Yau, On the Schrödinger equation and the eigenvalue problem, Commun. Math. Phys. 88 (1983) 309-318.

[20] E. Lieb, The number of bound states of one-body Schrodinger operators and the Weyl problem, Proc. Symp. Pure Math. 36 (1980) $241-252$.

[21] J. Descloux, M. Tolley, An accurate algorithm for computing the eigenvalues of a polygonal membrane, Comput. Methods Appl. Mech. Eng. 39 (1) (1983) 37-53. 